\title{
The Sound of Inattention: Predicting Mind Wandering with Automatically Derived Features of Instructor Speech
}

\author{
Ian Gliser ${ }^{1(\otimes)}$, Caitlin Mills ${ }^{1}$, Nigel Bosch ${ }^{2}$, Shelby Smith $^{1}$, Daniel Smilek ${ }^{3}$, \\ and Jeffrey D. Wammes ${ }^{4}$ \\ ${ }^{1}$ University of New Hampshire, Durham, NH, USA \\ ig1018@wildcats.unh. edu \\ 2 University of Illinois Urbana-Champaign, Champaign, IL, USA \\ 3 University of Waterloo, Waterloo, ON, Canada \\ 4 Queen's University, Kingston, ON, Canada
}

\begin{abstract}
Lecturing in a classroom environment is challenging - instructors are tasked with maintaining students' attention for extended periods of time while they are speaking. Previous work investigating the influence of speech on attention, however, has not yet been extended to instructor speech in live classroom lectures. In the current study, we automatically extracted acoustic features from live lectures to determine their association with rates of classroom mind-wandering (i.e., lack of student attention). Results indicated that five speech features reliably predicted classroom mind-wandering rates (Harmonics-to-Noise Ratio, Formant 1 Mean, Formant 2 Mean, Formant 3 Mean, and Jitter Standard Deviation). These speaker correlates of mind-wandering may be a foundation for developing a system to provide feedback in real-time for lecturers online and in the classroom. Such a system may prove to be highly beneficial in developing real-time tools to retain student attention, as well as informing other applications outside of the classroom.
\end{abstract}

Keywords: Mind-wandering $\cdot$ Attention $\cdot$ Acoustics $\cdot$ Speech $\cdot$ openSMILE

\section{Introduction}

In the classroom, lecturers are often faced with the challenging task of combatting frequent bouts of student inattention and disengagement. Such inattention often arises in the form of mind-wandering, defined here as thoughts unrelated to the task at hand (e.g., a classroom lecture $[31,32]$ ). When a student mind-wanders, they risk missing out on critical pieces of information and thus can develop an impoverished understanding of the learning material. It is therefore important to find ways to reduce the occurrence of mind-wandering and potentially mitigate its negative impact.

One way to minimize the potential negative influence of mind-wandering is by detecting and responding to it in real-time [20]. However, approaches to date have mostly focused on student-centered models of mind-wandering - where ongoing data specific to each learner (e.g., eye-gaze) are necessary to make predictions about whether or not 
they are currently mind-wandering. These attempts have been successful in laboratory contexts, but they are not currently scalable to entire classrooms.

Here we adopt an environment-centered model instead, by focusing on subtle naturalistic fluctuations in the learning environment (i.e., the instructor's speech). We test, for the first time, whether instructor speech patterns are related to classroom mind-wandering - potentially setting the foundation for the development environment-centered models of mind-wandering that can mitigate mind-wandering through scalable automated instructor feedback.

Environment-centered models seem based on the lessons we have already learned from laboratory cognitive psychology studies about when and why mind-wandering occurs. For example, mind-wandering tends to increase over the course of a task [33], and decrease when the task becomes more difficult (but see [13, 29]). Notably, even features like typeface seem to influence how often learners report mind-wandering: participants reported mind-wandering more often when reading a text in grey Comic Sans versus black Arial [11]. Although these studies demonstrate the potential malleability of mind-wandering, it is unclear if subtle environmental features (e.g., instructor behaviors, content changes, speech) may influence mind-wandering in live classrooms.

Here we directly examine how variations in the way information is transmitted through speech relates to students' attention in classroom contexts. This study builds on the environment-centered approach adopted by Bosch et al. [3], which examined how fluctuations in instructor movements were found to successfully predict classroom mind-wandering rates. Our specific focus on the instructor's speech fills an important gap in the literature, as very little research to date has been dedicated to quantifying and understanding how acoustical speech patterns influence student attention (e.g., rates of mind-wandering).

\subsection{Background Literature}

Acoustical features of speech have previously been linked to listener attention and information retention, albeit outside of the educational realm [4, 24]. For example, both the structure of speech (e.g., pitch contour and trajectory of source location) as well as prosodic quality (e.g., pitch and loudness) appear to reliably predict audience inattention $[5,10]$. The emotional tone conveyed through acoustical features also seems to be an important aspect of speech; for example, there are clearly dissociable processing patterns in the brain when people hear angry versus neutral prosody [26]. These studies, though not conducted in the context of a lecture, highlight the potential for acoustic-prosodic features to impact information processing - making it likely that mind-wandering may also be influenced.

Only a few studies have attempted to link acoustic features to mind-wandering specifically. Drummond \& Litman [6] asked students to read a paragraph about biology aloud and then perform a learning task (either self-explanation or paraphrasing). Periodically, they were probed to report how frequently they experienced off-task thoughts on a scale from 1 (all the time) to 7 (not at all) during the task. Students' responses were split into two categories, where 1-3 on the scale was "high" in zoning out, and 5-7 was "low" in zoning out. They trained a supervised machine learning model on the students" acoustic-prosodic features to classify low and high zone out, and achieved an accuracy 
of $64 \%$ in discriminating between the two. This study provides some evidence that individuals' tendencies to mind-wander are related to acoustic-prosodic cues (e.g., percent of silence, pitch, energy) of their own speech. It remains unclear, however, how such acoustic-prosodic features extracted from a speaker influence mind-wandering for listeners. Establishing associations between these speaker features and listener attention may have direct applications for an environment-centered feedback system in a classroom.

In the current study we sought to bridge the gap in our understanding of possible associations between features of speech and classroom attention. Our goals were (1) to provide a proof-of-concept method for automatically analyzing classroom speech features from low-cost audio recordings, and (2) to elucidate the relationship between acoustical speech features and mind-wandering in the classroom.

To tackle the first goal, we automatically extracted speech features from classroom lecture recordings using an open source software package called open Speech and Music Interpretation by Large-Space Extraction (openSMILE) [9]. We selected a popular feature set provided by openSMILE - the Geneva Minimalistic Acoustic Parameter Set [8]. We then identified and extracted a set of theoretically-relevant acoustic features from nine live classroom lecture recordings.

Next, as a step toward identifying key features to use in an environment-centered model of mind-wandering, we assessed the relation of these features to mind wandering. We focused on mind-wandering because it is consistently reported to be negatively associated with performance and comprehension in complex learning environments [19, 23, 31], including university lectures [34-36]. We aligned speech features in time with students' self-reported mind-wandering rates in order to probe this relationship. Below we describe our method for processing the audio recordings, how we arrived at a set of theoretically-relevant acoustic-prosodic variables, and how we tested for associations with mind wandering behavior.

\section{Method}

To address our two research goals, we collected data from multiple sources. As an overview, audio was extracted from low-cost video recordings of lectures, and students' attention was polled using a computer application during these same lectures. The two data channels were temporally aligned at 500-second intervals for analyses. Each stage is outlined in greater detail below.

\subsection{Classroom Audio and Self-reported Mind Wandering}

We extracted audio from recordings of nine different lectures at the University of Waterloo. These lectures were delivered by three different instructors (three lectures each) who were teaching undergraduate psychology courses. The lectures were delivered during normal classroom meeting times and with no manipulations or interference related to our experiments. The lectures took place in two similar classrooms, each with sloped, stadium-style seating and a stage with a podium for the teacher. The audio recording began at the same time as the lecture and lasted for the entirety of the class. For more details on data collection, please refer to Wammes et al. and Bosch et al. [3, 36]. 
Mind-wandering self-reports were collected from the students who participated in the study during the lecture $(\mathrm{N}=76)$. Students who agreed to participate in the experiment downloaded an application onto their laptop that administered pseudo-randomly scheduled thought probes throughout the lecture. Specifically, the occurrence of each thought probe notification was individually randomized, with the constraint that probes appeared no more than five times throughout the lecture with a range of 15 and 25 min between probes. When a thought probe was scheduled, a small window appeared in the bottom right corner of their computer screen. This prompted participants to introspect about their mental state just prior to the probe, and report their current degree of mind-wandering on a continuous scale ranging from Completely mind-wandering to Completely on task (reverse scored to correspond numbers between 0 and 1 , where higher values refer to more mind-wandering). They were informed that mind-wandering was defined as "thinking about unrelated concerns," and on task was defined as "thinking about the lecture."

\subsection{Audio Processing and Feature Extraction}

In order to avoid interference from speech unrelated to lecture delivery, we used Audacity software to trim each audio clip to only include the instructor's speech. Trimmed audio was then processed using openSMILE [10]. openSMILE is a flexible, open-source software package and audio toolkit capable of extracting a variety of different sound-based features, tailored for applications ranging from music to speech. The software extrapolates features based upon one's chosen configuration package and returns information about the occurrences of the selected features [10]. In this experiment, the configuration package was an implementation of the GeMAPS, a set of acoustic parameters based upon recent acoustical speech research [8]. GeMAPS was selected as the configuration for openSMILE due to its minimalistic approach to affect-oriented audio feature extraction. These parameters are Pitch, Jitter, Formant 1, 2, and 3 frequencies (F1, F2, and F3, respectively), F1 bandwidth, Shimmer, Loudness, Harmonics-to-noise-ratio (HNR), Alpha ratio, Spectral slope of $0-500 \mathrm{~Hz}$ and $500-1500 \mathrm{~Hz}, \mathrm{~F} 1, \mathrm{~F} 2$, and F3 relative energy, Harmonic difference H1-H2, and Harmonic difference H1-A3 [8].

Various relevant summary statistics of these basic parameters are also output by openSMILE. These include coefficient of variation (standard deviation normalized by the mean; SD) and mean for each parameter. For Loudness and Pitch, the following features were additionally included: $20^{\text {th }}$ percentile, $50^{\text {th }}$ percentile, $80^{\text {th }}$ percentile, the range of $20^{\text {th }}$ to $80^{\text {th }}$ percentile, as well as the mean and SD of the slope rising signal and slope falling signal. Lastly, the mean of Spectral slopes (from 0-500 Hz and 500$1500 \mathrm{~Hz}$ ), the Alpha Ratio, and the Hammarberg Index were included for each recording, resulting in 56 total features for analysis.

Following extraction of audio recordings from lectures and feature extraction, we identified a subset of these GeMAPS features (described in more detail below) for our analysis. 


\subsection{Selecting Acoustic Features}

We identified a set of theoretically-relevant acoustical characteristics based on previous literature. Specifically, we searched for features that have well-established relationships with psychologically-relevant constructs (see Table 1 for a full description of features and corresponding sources). Due to the lack of classroom-based investigations, the majority of literature review focused on studies examining how features of speech relate to attention and emotion, broadly conceived, in laboratory contexts. For example, emotion is considered to be a fundamental aspect of speech, as the delivery of emotional information is tied to inflection of the voice [21]. The following features were identified with the corresponding sources, as described in Table 1.

Table 1. Full list of selected acoustical speech features, description of selected features, rationale for selection and citations of relevant literature

Formant 0 (F0) Mean. This feature characterizes the fundamental frequency of voice, which is critical in driving inflection and linked to prosody [18, 22, 27]

Formant 1 (F1) Mean. The first harmonic formant is a determinant of prosodic quality, which drives speech reception [18, 26-27].

Formant 2 (F2) Mean. The second harmonic formant uniquely defines sounds of speech and is an acoustic correlate of resonance and clearness of speech [18, 25-27]

Formant 3 (F3) Mean. The third harmonic formant is present in vowel sounds and is fundamental to reception of clear speech [1, 18, 26-27]

F1 Bandwidth Mean. This is the region of frequency in which amplitudes differ by less than 3 decibels from the center frequency. It is a determinant of nasally/honky qualities of speech [17-18].

Loudness Mean. The average maximum volume of speech indicates more careful and precise speech and is correlated to confident speech as well as compliance [15, $18]$.

Loudness Standard Deviation. See above (Loudness Mean).

Jitter Standard Deviation. The standard deviation of pitch fluctuations is associated with trembling/tremorous voices, relating it to nervous voice [30].

Shimmer Mean. The average fluctuations of speech loudness are also associated with trembling/tremorous voices, relating it to nervous voice [30].

Voiced Segment Mean Length. The average length of discrete units in a stream of speech is a correlate of confident and compliant speech, indicative of precise and careful speech $[15,18]$.

Harmonics-to-Noise Ratio. This is the ratio of harmonic energy difference between the fundamental formant (F0), first harmonic (F1) and second harmonic (F2). This is a correlate of rough, uneven, and bumpy speech [7].

Hammarberg Index Mean. The difference in spectral energy between peaks in the $0.2 \mathrm{kHz}$ and $2.5 \mathrm{kHz}$ band [16] is a correlate of low percentile sadness and perceived attractiveness of the speaker $[15,18]$. 


\subsubsection{Formants}

Formants are descriptions of the high regions of spectral energy that occur in discrete regions of frequency. F1, 2 and 3 are necessary for synthesis of vowels in speech; additionally, the presence of $\mathrm{F} 3$ is required for interpretable speech [37]. F1 Bandwidth Mean also describes the degree to which speech is nasally and thus is included here (see Table 1).

\subsubsection{Voiced Segment Length, Loudness, Jitter and Shimmer}

Loudness of Speech is vital in a lecture due to its important role in conveying information to all members of the audience as well as its relation to confident and precise speech $[15,18]$.

Voiced Segment Length is defined as the length of discrete units in a stream of speech, which is measured by recording the average periods of uninterrupted speech. Similar to loudness of speech, voiced segment length is a correlate of confidence and precision in speech $[15,18]$.

Shimmer Mean is described as the occurrences of fluctuations in loudness of speech. Somewhat analogous, Jitter Standard Deviation is defined as the fluctuations in pitch. Both shimmer and jitter have been found to be correlates of trembling and nervous speech [30].

\subsubsection{Harmonics-to-Noise Ratio and Hammarberg Index}

Harmonics-to-Noise Ratio (HNR) is the ratio of harmonic energy: the difference between fundamental formant (F0), first formant (F1), and second formant (F2). Previous research has found HNR to be a correlate of rough, uneven, and bumpy speech.

\subsection{Predicting Student Mind-Wandering with Acoustic Features}

Speech features were processed and extrapolated from the audio recordings in $500 \mathrm{~s}$ epochs of time, whereas students' mind-wandering reports were sampled continuously throughout the lecture. To facilitate comparison between these two data channels, speech features were paired with mind-wandering reports within the same $500 \mathrm{~s}$ window. To accomplish this, mind wandering reports were aggregated across participants within each time window from which acoustic features were derived. This resulted in 72 time windows per class, which we used in the analyses below. The average rating of mindwandering (on a continuous scale between $0-1$, where higher values mean more mindwandering) was $.499(S D=.287)$.

Relationships between speech features and mind-wandering rates were assessed using linear mixed-effects models. We used the lme4 package in $\mathrm{R}$ [2]. All models included a random effect of class to control for within-class variability in baseline mindwandering. All models regressed mind-wandering on each acoustic feature of interest. We used restricted maximum likelihood estimation (REML) with unstructured covariance to avoid biasing the error variance. Tests of model significance were computed using a type II Wald chi-square test with a two-tailed $\alpha$ of .05 from the car package to take a conservative approach based on only estimates from the model [14]. 


\section{Results}

Descriptive statistics for each theoretically-relevant feature can be found in Table 2. Below we describe how each of these features related to classroom rates of mindwandering. Effect sizes (i.e., standardized regression coefficients) can be found in Table 3. We checked for normality of the residuals (i.e. an assumption for linear regressions), and the residuals displayed a normal distribution.

Table 2. Mean and standard deviation of speech features and mind wandering reports.

\begin{tabular}{lcc}
\hline Features & $\boldsymbol{M}$ & $\boldsymbol{S D}$ \\
\hline Formant 0 Mean & 28.3 & 2.04 \\
Formant 1 Mean & 638 & 55.8 \\
Formant 2 Mean & 1683 & 56.0 \\
Formant 3 Mean & 2750 & 59.0 \\
Formant 1 Bandwidth Mean & 1190 & 34.0 \\
Loudness Mean & .571 & .105 \\
Loudness Standard Deviation & .452 & .080 \\
Jitter Standard Deviation & 1.56 & .117 \\
Shimmer Mean & 1.40 & .027 \\
Voiced Segment Length Mean & .168 & .049 \\
Harmonics-to-Noise Ratio & 2.01 & 1.74 \\
Hammarberg Index Mean & .359 & .892 \\
\# of Mind Wandering in Epoch & 11.8 & 12.6 \\
\hline
\end{tabular}

\subsection{Formants}

Formant 0 Mean $(\beta=.047, p=.494)$ and Formant 1 Bandwidth Mean $(\beta=.102, p=$ .156) were not reliably related to mind-wandering. In contrast, Formant 1 Mean $(\beta=-$ $.236, p=.006)$, Formant 2 Mean $(\beta=-.188, p=.025)$ and Formant 3 Mean $(\beta=-$ $.239, p=.007)$ were all significantly negatively related to classroom of mind-wandering.

\subsection{Loudness, Voiced Segment Length, Jitter and Shimmer}

Mind-wandering was not significantly related to Loudness Mean $(\beta=-.094, p=.128)$ or Loudness Standard Deviation $(\beta=.024, p=.801)$. The same non-significant patterns were observed between mind-wandering and Voiced Segment Length Mean $(\beta=.056, p$ $=.482)$ and Shimmer Mean $(\beta=-.051, p=.367)$. However, Jitter Standard Deviation was significantly positively related to mind-wandering $(\beta=.097, p=.008)$. 
Table 3. Linear mixed effects models of relevant acoustical speech features. Standardized regression coefficients and $p$-values listed for each model with significant values $(p<.05)$ bolded.

\begin{tabular}{lcc}
\hline \multicolumn{1}{c}{ Features } & $\boldsymbol{\beta}$ & $\boldsymbol{p}$ \\
\hline Formant 0 Mean & .047 & .494 \\
Formant 1 Mean & -.236 & $\mathbf{. 0 0 6}$ \\
Formant 2 Mean & $\mathbf{- . 1 8 8}$ & $\mathbf{. 0 2 5}$ \\
Formant 3 Mean & $\mathbf{- . 2 3 9}$ & $\mathbf{. 0 0 7}$ \\
Formant 1 Bandwidth Mean & .102 & .156 \\
& & \\
Loudness Mean & -.094 & .128 \\
Loudness Standard Deviation & .024 & .801 \\
Jitter Standard Deviation & $\mathbf{. 0 9 7}$ & $\mathbf{. 0 0 8}$ \\
Shimmer Mean & -.051 & .367 \\
Voiced Segment Length Mean & .056 & .482 \\
& & \\
Harmonics-to-Noise Ratio &. $\mathbf{1 7 5}$ & $\mathbf{. 0 0 9}$ \\
Hammarberg Index Mean & .071 & .224 \\
\hline
\end{tabular}

\subsection{Harmonics-to-Noise Ratio and Hammarberg Index}

Harmonics-to-noise Ratio was significantly positively related to mind-wandering ( $\beta=$ $.175, p=.009)$, whereas Hammarberg Index Mean was not $(\beta=.071, p=.224)$.

\subsection{Summary of Results}

We show that subtle fluctuations in speech characteristics influence classroom mindwandering. Findings indicate that higher speech interpretability (higher values of Formant 1, Formant 2, and Formant 3 Mean), stability of pitch inflection (lower Jitter Standard Deviation, and the smoothness and evenness of speech (lower Harmonics-tonoise Ratio) were associated with lower rates of self-reported mind-wandering. This same pattern of results was unchanged when analyses were repeated with the number of mind-wandering reports as a control variable.

\section{Discussion}

To date, little research has been devoted to environment-centered models of mindwandering in classrooms. However, understanding how subtle variations in classroom lectures relate to student attention is important given that students frequently report mind-wandering while listening to lectures. We addressed this gap by assessing the relationship between acoustical speech features and mind-wandering in a classroom setting. 
We first detailed a method for automatically extracting a set of psychologically-relevant acoustical speech features from low-cost video recordings in live classrooms. We then related these features to students' self-reported mind-wandering across nine lectures. The data indicate that acoustical characteristics of the instructor's speech matter: we observed significant relationships between mind-wandering and Formant 1, Formant 2, and Formant 3 Means as well as Jitter Standard Deviation and Harmonics-to-noise Ratio. Of these features, Jitter Standard Deviation and Harmonics-to-noise Ratio were positively related to student mind-wandering, whereas Formants 1, 2, and 3 Means were negatively related to mind-wandering.

The negative relationships seen for Formant 1, Formant 2, and Formant 3 Means suggest that students paid more attention when speech was clearer. These three formants are associated with better speech clarity and negative correlates of raspy or hard-to-hear speech $[18,26,27]$. From a cognitive standpoint, mind-wandering may be more likely to occur when speech is less clear because discerning the content of the speech becomes more difficult; students may not have the cognitive resources available to match these increased task demands $[13,38]$.

The positive relationship observed for Jitter Standard Deviation provide some insight into the role of pitch changes in mind-wandering; that is, as the volatility of the instructor's pitch increased, students reported higher rates of mind-wandering. Prior work revealed an association between jitter and trembling or nervous speech [30]. Thus, mind-wandering occurrences may increase when nervousness becomes detectable in an instructor speech. This relationship may be due to how students interpret of the prosody speech; additional sub textual emotional information that is actually irrelevant to the lecture may influence their attention. A similar positive relationship was found for Harmonics-to-Noise Ratio Mean: mind wandering increased along with increased magnitude of Harmonics-to-Noise Ratio (i.e., rough, uneven speech [15, 18]). In the context of our findings, this suggests that mind-wandering is more likely to occur when the instructor's speech is more rough and uneven. Taken together, these finding highlight promising avenues for improving classroom lectures given that overall clarity of speech may be a simple way to increase student attention.

Our findings serve as a potential basis for an environment-centered system to address mind-wandering that does not require any intrusive or high-cost student measurements. Current developments for such a system are in their infancy and rarely explore raw audio data. For example, Schneider, Borner, Van Rosmalen \& Specht [28] developed a real-time feedback system which analyzes nonverbal and verbal behaviors and provides feedback to speakers. This system, while found to increase performance and confidence in speaking, focused on more basic features than the ones used here, such as arm-crossing and volume. This system seeks to ensure that fluid speech is maintained and does not currently incorporate potential predictors of listener attention such as mind-wandering. Our findings suggest that interventions may be effective by targeting acoustical characteristics of instructor speech to improve lecture delivery and reduce classroom mind-wandering.

Our results, when combined with other environment-centered features like movement [3], may provide a crucial step toward the development of a multi-modal feedback system where acoustic properties are considered along with motion, content, and other sources of classroom data. Future research in this area may benefit by repeating the experiment 
while controlling for lecture content, as content of the presented information is likely to influence off-task though. Next steps include integrating and testing automatically extracted acoustic features into a real-time system. Such a system would require the intake of data in a specified time window, buffering the data in order to allow time for openSMILE computation, then feeding the buffered data to openSMILE for computation and prediction of student attention. Finally, the system will need to return a report that is easily accessible and interpretable by the instructor. While these steps are substantive, they are all attainable and the efficacy of using both video and auditory features has been established. As these tools develop, they provide a promising direction for online real-time intervention in educational settings.

\section{References}

1. Barrichelo-Lindström, V., Behlau, M.: Resonant voice in acting students: perceptual and acoustic correlates of the trained Y-buzz by Lessac. J. Voice 23, 603-609. https://doi.org/10. 1016/j.jvoice.2007.12.001

2. Bates, D., Mächler, M., Bolker, B., Walker, S.: Fitting linear mixed-effects models using lme4. J. Stat. Softw. 67, 201-210 (2015). https://doi.org/10.18637/jss.v067.i01

3. Bosch, N., Mills, C., Wammes, J.D., Smilek, D.: Quantifying classroom instructor dynamics with computer vision. In: Penstein Rosé, C. et al. (eds.) AIED 2018. LNCS, vol. 10947. Springer, Heidelberg (2018). https://doi.org/10.1007/978-3-319-93843-1_3

4. Darwin, C.J., Hukin, R.W.: Effectiveness of spatial cues, prosody, and talker characteristics in selective attention. J. Acoust. Soc. Am. 107, 970-977 (2000). https://doi.org/10.1121/1. 428278

5. Ding, N., Simon, J.Z.: Cortical entrainment to continuous speech: functional roles and interpretations. Front. Hum. Neurosci. 8 (2014). http://doi.org/10.3389/fnhum.2014.00311

6. Drummond, J., Litman, D.: In the zone: towards detecting student zoning out using supervised machine learning. In: Aleven, V., Kay, J., Mostow, J. (eds.) ITS 2010. LNCS, vol. 6095, pp. 306-308. Springer, Heidelberg (2010). https://doi.org/10.1007/978-3-642-13437-1_53

7. Eskenazi, L., Childers, D.G., Hicks, D.M.: Acoustic correlates of vocal quality. J. Speech Hear. Res. 33, 298-306 (1990). https://doi.org/10.1044/jshr.3302.298

8. Eyben, F., Scherer, K.R., Schuller, B.W., et al. The Geneva Minimalistic Acoustic Parameter Set (GeMAPS) for voice research and affective computing. IEEE Trans. Affect. Comput. 7, 190-202 (2016). https://doi.org/10.1109/TAFFC.2015.2457417

9. Eyben, F., Weninger, F., Groß, F., Schuller, B.: Recent developments in openSMILE, the Munich open-source multimedia feature extractor categories and subject descriptors. In: Proc 21st ACM International Conference Multimedia, pp. 835-838 (2013). https://doi.org/10.1145/ 2502081.2502224

10. Eyben, F., Wöllmer, M., Schuller, B.B., et al.: OPENSMILE: open-source media interpretation by large feature-space extraction. In: MM 2010 - Proceedings of ACM Multimedia 2010 International Conference, pp. 1-65 (2015). https://doi.org/10.1145/1873951.1874246

11. Faber, M., Mills, C., Kopp, K., D’Mello, S.: The effect of disfluency on mind wandering during text comprehension. Psychon. Bull. Rev. 24, 914-919 (2017). https://doi.org/10.3758/ s13423-016-1153-z

12. Faber, M., Radvansky, G.A., D’Mello, S.K.: Driven to distraction: a lack of change gives rise to mind wandering. Cognition 173, 133-137 (2018). https://doi.org/10.1016/j.cognition. 2018.01.007

13. Feng, S., D’Mello, S., Graesser, A.C.: Mind wandering while reading easy and difficult texts. Psychon. Bull. Rev. 20, 586-592 (2013). https://doi.org/10.3758/s13423-012-0367-y 
14. Fox, J., Friendly, M., Weisberg, S.: Hypothesis tests for multivariate linear models using the car package. R J. 5(1), 39-52 (2013)

15. Fernandez Gallardo, L., Weiss, B.: Perceived interpersonal speaker attributes and their acoustic features. In: Proceedings of the Phonetic \& Phonologie, pp. 61-64 (2017)

16. Hammarberg, B., Fritzell, B., Gaufin, J., et al.: Perceptual and acoustic correlates of abnormal voice qualities. Acta Otolaryngol. 90, 441-451 (1980). https://doi.org/10.3109/000164880 09131746

17. Lee, A.S.Y., Ciocca, V., Whitehill, T.L.: Acoustic correlates of hypernasality. Clin. Linguist. Phonetics 17, 259-264 (2003). https://doi.org/10.1080/0269920031000080091

18. Memon, S.A.: Acoustic Correlates of the Voice Qualifiers Summarizing the Perception of Qatar on Twitter View project Algorithms for Speaker Profiling (2018). https://doi.org/10. 13140/RG.2.2.24759.57764

19. Mills, C., Graesser, A., Risko, E.F., D’Mello, S.K.: Cognitive coupling during reading. J. Exp. Psychol. Gen. 146, 872-883 (2017). https://doi.org/10.1037/xge0000309

20. Mills, C., Gregg, J., Bixler, R., D’Mello, S.K.: Eye-Mind reader: an intelligent reading interface that promotes long-term comprehension by detecting and responding to mind wandering. Hum.-Comput. Interact. 1-27 (2020). https://doi.org/10.1080/07370024.2020.1716762

21. Murray, I.R., Arnott, J.L.: Toward the simulation of emotion in synthetic speech: a review of the literature on human vocal emotion. J. Acoust. Soc. Am. 93, 1097-1108 (1993). https:// doi.org/10.1121/1.405558

22. Paeschke, A., Kienast, M., Sendlmeier, W.F., Berlin, T.U.: F0contours in emotional speech. In: Proceedings of the 14th International Congress of Phonetic Sciences (1999)

23. Randall, J.G., Oswald, F.L., Beier, M.E.: Mind-wandering, cognition, and performance: a theory-driven meta-analysis of attention regulation. Psychol. Bull. 140, 1411-1431 (2014). https://doi.org/10.1037/a0037428

24. Rimmele, J.M., Zion Golumbic, E., Schröger, E., Poeppel, D.: The effects of selective attention and speech acoustics on neural speech-tracking in a multi-talker scene. Cortex 68, 144-154 (2015). https://doi.org/10.1016/j.cortex.2014.12.014

25. Robb, M., Blomgren, M.: Analysis of F2 transitions in the speech of stutterers and nonstutterers. J. Fluency Disord. 22, 1-16 (1997). https://doi.org/10.1016/S0094-730X(96)000 16-2

26. Sander, D., Grandjean, D., Pourtois, G., et al.: Emotion and attention interactions in social cognition: brain regions involved in processing anger prosody. Neuroimage 28, 848-858 (2005). https://doi.org/10.1016/j.neuroimage.2005.06.023

27. Scherer, K.R., London, H., Wolf, J.J.: The voice of confidence: paralinguistic cues and audience evaluation. J. Res. Pers. 7, 31-44 (1973). https://doi.org/10.1016/0092-6566(73)900 $30-5$

28. Schneider, J., Borner, D., Van Rosmalen, P., Specht, M.: Can you help me with my pitch? Studying a tool for real-time automated feedback. IEEE Trans. Learn. Technol. 9, 318-327 (2016). https://doi.org/10.1109/TLT.2016.2627043

29. Seli, P., Konishi, M., Risko, E.F., Smilek, D.: The role of task difficulty in theoretical accounts of mind wandering. Conscious Cogn. 65, 255-262 (2018). https://doi.org/10.1016/j.concog. 2018.08.005

30. Shao, J., MacCallum, J.K., Zhang, Y., et al.: Acoustic analysis of the tremulous voice: assessing the utility of the correlation dimension and perturbation parameters. J. Commun. Disord. 43, 35-44 (2010). https://doi.org/10.1016/j.jcomdis.2009.09.001

31. Smallwood, J., Fishman, D.J., Schooler, J.W.: Counting the cost of an absent mind: mind wandering as an underrecognized influence on educational performance. Psychon. Bull. Rev. 230-236 (2007). https://doi.org/10.3758/bf03194057

32. Smallwood, J., Schooler, J.W.: The restless mind. Psychol. Bull. 132, 946-958 (2006). https:// doi.org/10.1037/0033-2909.132.6.946 
33. Thomson, D.R., Seli, P., Besner, D., Smilek, D.: On the link between mind wandering and task performance over time. Conscious Cogn. 27, 14-26 (2014). https://doi.org/10.1016/j.con $\operatorname{cog} .2014 .04 .001$

34. Wammes, J.D., Seli, P., Cheyne, J.A., et al.: Mind wandering during lectures II: relation to academic performance. Scholarsh. Teach. Learn. Psychol. 2, 33-48 (2016). https://doi.org/ $10.1037 /$ st10000055

35. Wammes, J.D., Smilek, D.: Examining the influence of lecture format on degree of mind wandering. J. Appl. Res. Mem. Cogn. 6, 174-184 (2017). https://doi.org/10.1016/j.jarmac. 2017.01.015

36. Wammes, J.D., Ralph, B.C.W., Mills, C., et al.: Disengagement during lectures: media multitasking and mind wandering in university classrooms. Comput. Educ. (2019). https://doi. org/10.1016/j.compedu.2018.12.007

37. Weiss, B., Burkhardt, F.: Voice attributes affecting likability perception. In: Proceedings of 11th Annual Conference International Speech Communication Association INTERSPEECH 2010 (2010)

38. $\mathrm{Xu}, \mathrm{J} .$, Metcalfe, J.: Studying in the region of proximal learning reduces mind wandering. Mem. Cognit. 44, 681-695 (2016). https://doi.org/10.3758/s13421-016-0589-8 